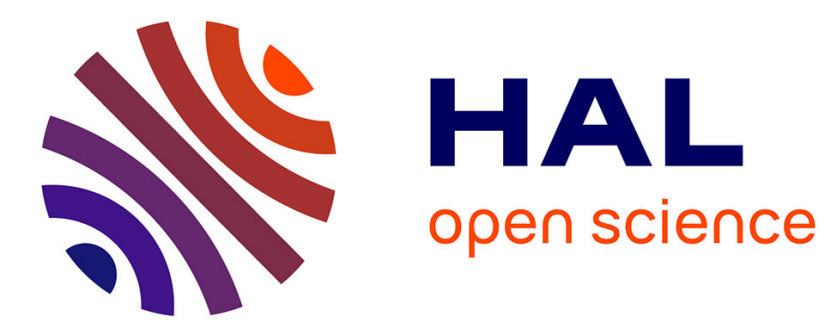

\title{
Risk communication: the European Commission TSE Roadmap Model for policy relaxation
}

\author{
Kerstin Dressel, Wim van Wassenhove, Alice Perazzini, Giuseppe Ru
}

\section{To cite this version:}

Kerstin Dressel, Wim van Wassenhove, Alice Perazzini, Giuseppe Ru. Risk communication: the European Commission TSE Roadmap Model for policy relaxation. International Journal of Risk Assessment and Management, 2012, 16 (4), p. 213-226. hal-00757092

HAL Id: hal-00757092

https:/ /hal-mines-paristech.archives-ouvertes.fr/hal-00757092

Submitted on 17 May 2013

HAL is a multi-disciplinary open access archive for the deposit and dissemination of scientific research documents, whether they are published or not. The documents may come from teaching and research institutions in France or abroad, or from public or private research centers.
L'archive ouverte pluridisciplinaire HAL, est destinée au dépôt et à la diffusion de documents scientifiques de niveau recherche, publiés ou non, émanant des établissements d'enseignement et de recherche français ou étrangers, des laboratoires publics ou privés. 


\title{
Risk Communication: The European Commission TSE Roadmap Model for Policy Relaxation
}

\begin{abstract}
:
The Bovine Spongiform Encephalopathy (BSE) crisis forced the European Commission to impose strict regulations, the most significant and overarching of which was Regulation (EC) No 999/2001. These regulations were responsible for the abatement of the BSE epidemic in Europe in the years that followed.

The 'Transmissible Spongiform Encephalopathy (TSE) Roadmap' was published by the European Commission several years later, on 15th July 2005. This Roadmap proposed the relaxation of BSE measures in the short, medium and long term. It was seen as a proactive way to prepare for the implementation of new regulations and in terms of risk communication, was a very interesting approach.

This paper presents the "roadmap model" as an effective tool for risk communication. It proposes several recommendations to help decision-makers in the difficult task of risk communication and establishes some conditions for generating public trust.
\end{abstract}

Keywords: risk communication, risk perception, risk management, policy relaxation, BSE crisis, TSE Roadmap, trust.

Dr. Wim VAN WASSENHOVE

Crisis and Risk Research Centre (CRC)

Mines Paris Tech

Rue Claude Daunesse BP 207

Sophia-Antipolis, 06904, France

wim.van_wassenhove@mines-paristech.fr

Dr. Kerstin DRESSEL

South German Institute of

Empirical Social Research (sine-Institute) e.V.

Lilienstr. 3

D-81669 München, Germany

$\underline{\text { Kerstin.Dressel@sine-institut.de }}$

Dr. Alice PERAZZINI

Italian Reference Centre for

Animal TSE (CEA)

Via Bologna 148

Torino, I- 10154, Italy

Alice.Perazzini@izsto.it

Dr. Giuseppe RU

Italian Reference Centre for

Animal TSE (CEA)

Via Bologna 148

Torino, I- 10154, Italy

Guiseppe.ru@izsto.it 


\section{Introduction}

Risk regulation is largely the result of a reaction to catastrophic events by national governments or at the European Union (EU) level. An example at EU level is the SEVESO directive (96/82/CE), and in France, the regulations drawn up in reaction to the AZF catastrophe at Toulouse in 2001 (law $\mathrm{n}^{\circ} 2003-699$ of $30^{\text {th }} \mathrm{July,} \mathrm{2003).}$

The BSE crisis formed part of this pattern of what can be termed 'aftermath regulations'. In the 1980s Bovine Spongiform Encephalopathy (BSE, mad cow disease), a fatal neurodegenerative disease, was the source of an epidemic in cattle in the United Kingdom (UK). In 1996, a worldwide alert was raised when the BSE agent was found to be transmissible to humans and the cause a new type of neurological disease, variant CreutzfeldtJakob disease (vCJD).

Since the European Community was founded, no debate has had such an impact on the daily life of individuals as that of BSE. In 1997 an ad hoc inquiry by the European Parliament made a devastating appraisal of the handling of the BSE crisis by both the UK government and the European Commission. The report of the inquiry emphasised that the damage caused by the BSE crisis among the general public should not be underestimated. It made particular reference to the fall in consumer confidence in food chain security, and pointed out that the final number of deaths remained unknown [European Parliament, 1997; Wynne \& Dressel, 2001].

The reaction of the European Commission was to implement strict regulations. The most significant and overarching of these was Regulation (EC) No 999/2001. Released on $22^{\text {nd }}$ May, 2001, it provided instructions for the prevention, control and eradication of certain Transmissible Spongiform Encephalopathies (TSEs). The BSE figures dropped sharply following the implementation (in the EU and elsewhere) of these rigorous, science-based control measures and it seems that they have succeeded in controlling the BSE epidemic, which has been in continuous decline in recent years.

When it became clear that the spread of BSE could no longer be called an epidemic, there was huge pressure from stakeholders to lift some of the more expensive measures ${ }^{1}$. This led the European Commission to publish the TSE Roadmap [European Commission, 2005]. The Roadmap was a 24-page strategy paper, which provided an outline of potential future changes to EU measures on BSE both in the short and medium term (2005-2009), and in the long term (2009-2014). At the time of writing, most of the short and medium term measures have already been adopted by the Commission.

This was a proactive way to approach to the preparation of new regulations and, from the point of view of risk communication, a very interesting development. Unlike Green or White Papers, the Roadmap was a short document that contained all the relevant (scientific and legal) information. It could be considered as a catalogue of options that were open to further discussion and invited everyone affected by the topic to participate in the discussion process.

This article therefore investigates the roadmap model as a new form of risk communication and as a means of initiating policy relaxation. Using the TSE Roadmap as an example, it discusses risk communication, perception and management. The first part describes an experimental investigation into stakeholders' risk perception. The second part presents some theoretical aspects of risk management, communication and perception. The third part

\footnotetext{
${ }^{1}$ To illustrate this point, in 2002 the cost of finding one positive case in the healthy slaughter surveillance stream (cattle aged 30-35 months) was 302 million euros [European Commission, 2005].
} 
discusses the potential contribution of the roadmap model to these three issues, and makes some recommendations with respect to risk communication.

\section{An experimental investigation}

This experimental study ${ }^{2}$ brought together four groups of stakeholders ${ }^{3}$ in five EU Member States $^{4}$ and at the European level in order to gather their perceptions of the TSE Roadmap and its implications for consumer protection. The objectives were: (1) to assess the risk perceptions of the four groups of stakeholders with respect to the relaxation of BSE measures, (2) to identify and to describe risk communication practices and strategies in dealing with the TSE Roadmap, and (3) to provide the European Commission and risk regulators in Member States with recommendations for communicating the implications of the TSE Roadmap [Dressel et al., 2009].

\section{Method}

A qualitative approach was chosen for this comparative social science study, which used indepth, semi-structured interviews ${ }^{5}$ [Boyce \& Neale, 2006], an analysis of the literature and Internet research. The decision was made to focus on specific stakeholder groups, who were considered to be representative of a broader public, as it was considered unlikely that the general public would be aware of the TSE Roadmap and its contents. The selected stakeholders could be reasonably assumed to be far more aware of the Roadmap and globally representative of the general public, particularly as some of the stakeholders interviewed belonged to consumer organizations. The decision to exclude the general public was validated by the results of the study, which showed that even some of the selected stakeholder groups were unaware of the Roadmap. It is therefore unlikely that members of the general public would have been able to usefully comment on its technical content without first having it explained to them in detail (a process which would have been very time consuming).

The groups of stakeholders selected to participate in the study (particularly consumer organisations) played an important role in informing both the public and the media. The approach taken by the Roadmap was to use these stakeholders as communication channels. Therefore, although substantially a technical document, the Roadmap had to be written in language that could still be understood by stakeholders (who participated in the decisionmaking process) and who had the subsequent responsibility for transmitting the message to the general public [Ru et al, 2010],[Dressel et al., 2011].

\footnotetext{
${ }^{2}$ This study was supported by a European Commission grant awarded to the Neuroprion Network of Excellence (FOOD-CT-2004-506579).

${ }^{3}$ The four stakeholder groups were: risk managers (i.e. governmental bodies responsible for TSE risk regulation such as ministries of health and agriculture and their advisory agencies), farmers' associations, consumer associations and the food industry (the food and meat industry, excluding the feed industry).

${ }^{4}$ The five selected countries were France, Italy, the United Kingdom, Germany and Belgium. This choice was motivated by the relevance of the countries involved and the nationality of the study's participants.

${ }^{5}$ In total 46 interviews were conducted. Participants were asked 30 main questions about their risk perception of TSEs, existing TSE risk regulations, risk communication and other questions related to stakeholder involvement in public policy-making. The interview time varied considerably, from 35 minutes to several hours with a mean of one hour.
} 


\section{Results}

The results of the study showed no distinguishable differences between stakeholders in TSE risk perception. All stakeholder groups in all countries agreed that the risk from BSE and other TSEs had diminished. This situation was ascribed to the effective risk management measures taken on a European basis. All interviewees shared the view that the TSE risk was clearly in decline and the overall perception was that the level of both BSE and TSE risk was low.

Overall, the study concluded that BSE was no longer a hot topic. This was reflected by the low profile of the subject in the media, which since 2007 had received little attention across the EU as a whole. The regulatory measures taken in 2000 were generally considered by stakeholders as drastic and severe, but extremely necessary at the time, both to reduce the risk and to rebuild consumer confidence and trust in the agricultural and food sector [Ru et al, 2010], [Dressel et al., 2011]. [Van Wassenhove et al., 2011].

This corresponds to the idea of 'out of sight, out of mind'. However, Slovic et al. [1982] demonstrated that communication of risk with a very low probability has the counterproductive effect of sharpening perception of that risk [in Kermisch, 2010]. This point should be kept in mind when communicating risk. At the same time, a paternalistic approach which sees the population as needing to be educated about risk through a one-way communication process does not help either to establish a dialogue or engage actors in risk decisions. Risk perception and risk communication are far more complex than that, and the BSE case is a good example.

Another finding of the study was that in all five countries the media provided hardly any information on TSEs. This emphasised the fact that risk managers and decision-makers cannot rely on the media to inform the public. Italian consumers had repeatedly asked for more information from their health authorities, and not just in the aftermath of the emergency. In Belgium, stakeholders commented that although the decisions of the European Commission were published, no details were provided of how those decisions were reached (e.g. assumptions, alternatives). In France, stakeholders had asked for more effective communication as a tool to regain consumer confidence and trust in food safety. However, in the UK, all stakeholders praised the communication of TSE risk by national official bodies (the Food Standards Agency) when compared to past performance. This is possibly due to the severe effect of the BSE crisis in the UK. As a result, these bodies restructured themselves in order to communicate more effectively [Van Wassenhove et al., 2011].

The study showed that the European Commission communicates essentially to governmental bodies, federations and private organisations at the European level. These organisations are in turn expected to communicate the information to the general public and federations at Member State level. However, this cascading of information is not always an effective way to reach national populations. As this study showed, the information in the Roadmap was not distributed to all national stakeholders. This raises the question of what is, what should be, and what could be the role of roadmaps (the TSE Roadmap in particular) in the EU's decision-making and communications strategy, and whether recommendations for the EU's risk communication strategy could be more precise. Any recommendations could be extrapolated to national government risk communication, and potentially to risk communication within companies.

The following section discusses some theoretical aspects of risk management, communication and associated risk perception. 


\section{Risk management, communication and perception}

In general, risk management, risk communication and risk perception are three interacting processes. Risk management measures should be communicated to the general public and as a result influence their risk perception. Interest in public risk perception has increased considerably since the 1980 s and is a phenomenon common to all industrialised countries. Increased interest in risk perception among the general public is frequently interpreted as an indicator of a modern-day crisis whereby society, faced with unintended side effects and unexpected fall-outs, increasingly questions the abilities of risk managers [Beck, 1986; Adam, 1995]. The BSE crisis is one example of this.

Risk managers have been obliged to act. They have been confronted by scandals, implementation deficiencies and public surveys in which the credibility of political decisionmakers, as well as government institutions, has been increasingly called into question. Farreaching decisions, with potentially negative consequences for citizens can only meet with social acceptance if those affected are involved in the process and their views are taken into account. Against this background, research on risk perception among the populace has become increasingly relevant. However, the point should be made that although in theory risk managers have become dramatically more aware of the importance of risk perception research, in practice the results of this research are neither used in a systematic and consistent manner, nor are they systematically tied into the risk management decision-making process [Dowler et al., 2006; Renn \& Benighaus, 2006].

There are a number of approaches that can be used to investigate social risk perception. They tend to be inspired by social psychology, cognitive psychology, anthropology or sociology and are orientated accordingly. To give some examples, Luhmann states that risk perception is the result of a process of social communication [Luhmann, 1986] and Slovic says that there is no such thing as 'real risk' or 'objective risk' [Slovic, 1992]. Risk appears to be a (social) construct in which communication plays an important role. Effective risk management and subsequent risk communication should be readily understandable by members of the public. This creates trust in risk managers, who must reconstruct and respond to the public's idea of what is perceived as risk.

Different groups have different perceptions of risk. Experts do not perceive risk in the same way as non-experts. Experts are guided by the available scientific and technological knowledge base (the so-called facts) in evaluating a risk. The public, on the other hand, are only marginally informed by these facts (in addition, the general public does not have adequate knowledge to fully understand them). These different bases for evaluation mean that risk perception by experts is usually termed risk assessment rather than risk perception [Renn \& Benighaus, 2006]. Last but not least, the media play a decisive role in what the public perceives as risk. A 2006 study on BSE demonstrated that the populations of Germany, Finland, Italy and the UK have different risk conceptualizations of BSE and that these differences are due to the influence of their national media [Bauer et al., 2006].

Therefore, in order to facilitate effective risk communication it is essential to have an improved understanding of risk perception. In this regard, risk communication is seen as the key to closing the gap between the risk assessment of experts and the general public's perception of risk, thereby arriving at a more effective risk management strategy and improved regulation [Renn \& Benighaus, 2006].

\section{The STARC project}

Other studies have discussed risk communication and its pitfalls. The EU-funded project 'STAkeholders in Risk Communication' (STARC) explicitly dealt with the role of risk 
communication and how to make the risk decision-making process more socially robust [Dien et al., 2006]. It made several recommendations:

- Improve the understandability of scientific reports through co-operation with journalists.

Frequently, insufficient effort has been made to make information more readily understandable by the general public. Risk experts should be proactive in initiating efforts to co-operate with science journalists in order to arrive at a common language.

- Make openness of the discussion process a precondition.

The public should be explicitly included in the risk management process in order to achieve a broader and more socially robust acceptance of decisions on how to manage a given risk.

- Establish ad hoc forums.

Ad hoc or temporary forums for discussion of risk issues should be established, preferably through a physical presence and/or on the Internet.

- Avoid a situation where professional PR becomes counter-productive for risk communication.

Public relations (PR) departments can be very useful. However, aggressive PR and marketing methods are counter-productive and may only raise suspicions.

- Make transparency a top priority in risk communication.

Transparency means being able to trace how authorities arrive at their decisions (a description of the criteria used for risk assessments, the limitations of knowledge, conditions for authorisation, etc.).

- Be aware of the importance of correct timing.

It is absolutely crucial to begin risk communication very early in order to avoid public concern. Furthermore, well-timed and intensive risk communication should support the public in their understanding of, and comment upon the issues.

- Remember that independent experts are considered trustworthy.

Independent experts are recognised by public authorities as well as industry and NGOs as important participants in the process of finding an effective response and solution to an ambiguous risk situation. Ideally, such experts are neither public sector employees, nor employed or funded by industry, nor members of an NGO. Ideally, independent experts are from academia. Experts from abroad are also often seen as being independent.

This section has addressed the theoretical background to risk management, risk communication and risk perception. The following section discusses the potential contribution of the roadmap model to these three issues.

\section{The roadmap model}

This section discusses the BSE risk communication approach and the potential contribution of the roadmap model to risk communication and building the trust of the general public in the decision-making process.

\section{The TSE Roadmap}

The TSE Roadmap is an excellent example of an appropriate way to arrive at robust decisions via consultation. The vast majority of the stakeholders interviewed in the BSE study [Van 
Wassenhove et al., 2011] found the timeframes (short, medium and long-term objectives) given in the TSE Roadmap useful, as they enabled work to be planned. As control measures were eased it was vital to control compliance and to ensure that any breaches were carefully and immediately reported. Covering-up simply created public mistrust.

\section{Language}

Stakeholders wanted to see the science behind any decisions related to amendments in TSE regulation. The science had to be explained in jargon-free language that stakeholders could understand (and communicate to the general public). There was therefore a tension between a scientific discussion of the background to risk management decisions and the communication of general ideas in understandable language. The final form of the TSE Roadmap was a widely accepted compromise between technical data and general ideas and was understood by almost all stakeholder groups with expert knowledge.

However, the TSE Roadmap could not be understood by the general public. Although it was clearly important to communicate in plain, simple terms, the TSE Roadmap was technical and technical language had to be used. Nevertheless, it was imperative that it was clearly understood by stakeholders, as these stakeholders played a crucial role in transmitting the information in a suitable form to the general public. One of the authors of this article has firsthand experience of this, having previously worked for a national food industry federation. The media know, and rely on the information channels provided by stakeholders to inform the general public. It is therefore clear that the various stakeholders groups (in particular consumer organisations) play an important role in informing both the public and the media.

\section{Dissemination}

However, not all stakeholders affected by the TSE Roadmap were actually aware of its existence! The most likely explanation for this is that not all representatives of stakeholder groups at European level (who interact with the European Commission in Brussels) forward the information to their colleagues at the national level (as discussed earlier). The Commission should consider effective ways to make sure that information is equally distributed to all concerned stakeholders at the Member State level. To achieve this, the Commission should identify communication channels and target organisations at the Member State level. This point demonstrates that effective risk communication not only involves writing a document with the right information in the right way, but also requires that close attention be paid to the distribution of the document.

The roadmap model, with appropriate language and science-based content, is an excellent tool for risk communication of the decision-making process. It is a tool that can, and must, be transposed to other domains. At the same time, effective communication and feedback channels must be identified to facilitate distribution of the message at Member State level. The principal way to improve the roadmap model is to ensure that these communication channels exist and are open.

The TSE Roadmap was a key element in risk perception as it reinforced trust in authorities and regulators as will be demonstrated in the next section.

\section{Building public trust}

Trust is the key to successful risk communication and plays a key role in risk regulation. Löfstedt states that without public trust in authorities/regulators, it is very difficult to put 
together a successful risk communication strategy and that there is a direct relationship between high public trust in authority and low perceived risk and vice versa. Indeed, it is possible to communicate issues of high uncertainty in a top-down fashion when the public trusts authorities/regulators [Löfstedt, 2003].

This suggests that the more pronounced the uncertainty (knowledge gap), the greater the need to establish a firm basis for trust. The objective of effective risk communication is to create the necessary conditions for a culture of trust across all levels. This cannot be a unilateral act; a culture of trust, under the conditions discussed above, can only be established using a symmetrical approach. Transparency, dialogue, participation and fairness must be given the highest priority [Maxwell, 2004]. A simple instrumental interpretation of risk communication as a means to create public trust is insufficient [PABE, 2001]. The best way to build public trust is as a by-product - by ensuring that procedures truly involve the public in decisionmaking [Trettin \& Musham, 2000]. Risk communication has to address knowledge gaps and uncertainties. In times of systemic risk where uncertainties are high, values are in dispute and urgent decisions must be taken, there is no such thing as zero-risk or absolute safety [Funtowicz \& Ravetz, 1993]. The aim of risk communication is not to downplay residual knowledge gaps and uncertainties, but instead to adequately address the limits of knowledge and the risk potential. It is also to increase and share knowledge among stakeholders and to make those concerned aware of residual risks in order to arrive at a decision. An example of this is the recommendations of the STARC project (see above), which aim to make the risk decision-making process more socially robust.

The roadmap model, in its improved form, is a tool in the risk decision-making and communication process that gives stakeholders (and hence, the broader public) an opportunity to be included. Greater public trust is a highly sought after by-product. Transparent decisionmaking processes, communicating in plain and simple language, stakeholder involvement and appropriate feedback channels are important factors in creating conditions that generate public trust as a by-product (Figure 1). The roadmap is an artefact that creates the opportunity to communicate and to involve stakeholders in the decision-making process, and thus, through its transparency, creates trust.

\section{Insert Figure 1 here}

\section{Recommendations}

This section describes recommendations for risk communication as part of a decision-making process that aims to obtain robust social acceptance and trust. These processes are interdependent and must be understood together. The recommendations are based on an analysis of stakeholders' interviews in the TSE Roadmap study [Dressel et al., 2009] and the STARC recommendations [Dien et al., 2006].

Sound scientific knowledge is a prerequisite for decision-making. In the TSE Roadmap study, all stakeholders in all of the examined countries emphasized that measures will be accepted when they are based on sound scientific knowledge and developed using a scientific risk assessment. It should be noted here that it is as important to report what is not known, as it is to report what is known, i.e. the limits and limitations of current scientific knowledge.

Communicate in plain, simple language. In general, risk reporting should be factual, straightforward and appropriate. In order to communicate to stakeholders and the public, it is imperative that messages should be understandable and describe the risk in simple, clear and 
honest terms. Technical and scientific jargon should be avoided. Even in scientific domains that have their own very sophisticated and less accessible languages (such as basic research) it is possible to find words that can be understood by non-experts. The goal for risk communication should be the formulation of scientifically correct messages, understandable by the general public. The UK Food Standards Agency provide an excellent example of how to communicate TSE matters effectively to the public and could be considered as a role model for TSE risk communication. Co-operation with science journalists may also be helpful. However, aggressive professional PR may be counterproductive.

Make the decision-making process transparent. Transparency, openness and good timing are important factors to consider in risk communication. The TSE Roadmap study found great interest among stakeholders in making the actual decision-making process as transparent as possible. The first step towards this is to report what is not known, as well as what is known about the topic. The next step should be to outline what options were considered (in terms of social and economic risks), to report what cost-benefit assumptions were made and why they led to certain conclusions and to conclude with an explanation of why particular decisions were taken while others were not.

Involve stakeholders in decision-making. In order to arrive at socially robust decision-making, decision-makers have to make sure that they are aware of the viewpoints of all the diverse, relevant stakeholders (a bottom-up information flow). Therefore, provision should be made, not only for stakeholders to make their views known, but also for them to see that their ideas have been incorporated into the decision-making process. Working groups, ad hoc forums and regular consultation are potential ways in which stakeholders can be involved in the decisionmaking process. It was clear from the TSE Roadmap study that in those countries where public involvement and consultation in a transparent decision-making process was most advanced (Belgium and the UK) stakeholder approval of risk management was most noticeable. The opposite is also true: the more stakeholders had to ask for greater participation and communication, the less their approval of, and trust in public policy-making on TSEs. It was significant that a request not to patronize the public emerged in the UK study.

Create new alliances for risk communication. In the TSE Roadmap study, Italian stakeholders pointed out the weakness of leaving risk communication exclusively to the media, who are not interested in providing a continuous and evolving stream of serious information when they can instead report on a constant flow of emerging scandals. A finding that emerged from German respondents was that improved co-operation between risk assessment bodies and consumer associations in risk communication could constitute a win-win situation for everyone in the field of public health. Sound scientific knowledge on the one hand, and consumer trust on the other, can together contribute to improving the public's receptiveness to risk communication messages and could, therefore, lead to a more risk-informed and riskmature citizen. Such new alliances are of course conceivable for other stakeholder groups.

Link risk assessment and risk management. French stakeholders in the TSE Roadmap study raised concerns that separating the activities of risk assessment and risk management might lead to a situation where neither social and economic considerations nor the results of costbenefit analyses were adequately addressed. This was seen as being due to the fact that they seem neither to be the responsibility of risk assessors, nor risk managers. French participants therefore asked for another round in the risk decision-making process where these issues are explicitly discussed and taken into account in the development of policy. 
Establish effective communication channels. Once decisions have been taken, they have to be communicated via the appropriate channels both to the stakeholders concerned and to the broader public (a top-down information flow). It is clear that the European Commission cannot simply rely upon the media to take up the subject and report it responsibly. Instead, it must identify reliable and efficient structures and organizations in Member States that are able to communicate the risk message appropriately and objectively. If this cannot be adequately achieved, the Commission should consider working together with the particular Member State to establish such an organization.

No-risk messages are not required. Whereas risk managers and some other stakeholders in the TSE Roadmap study expressed a view that the public would ask for 'zero-risk' and 'no-risk' messages, the contrary is in fact the case. There was no evidence in any of the examined countries of such a request (e.g. from consumers). On the contrary, there was an emphasis on the fact that there is no such thing as 'zero-risk' in life. We therefore conclude that there is little merit in the idea that 'no-risk' messages are necessary to maintain public confidence. This of course assumes that risk managers take their responsibility as a public sentinel seriously.

\section{Conclusion}

This article described the TSE Roadmap as a communication model and proposed risk communication recommendations, in this case, specially for policy relaxation. The benefits of an improved roadmap as a new communication strategy are obvious. Unlike Green or White Papers, the roadmap is a short document that contains all the relevant (scientific and legal) information. It can be considered as a catalogue of options that are open to further discussion and invite everyone affected by the issue to participate in the discussion process. The consultation process is organised and takes place within a given timeframe. Finally, the process is made transparent by making all documents available to the public on the Internet. However, despite these efforts, not all the stakeholders who should have been involved in the TSE Roadmap were actually aware of its existence. It is therefore clear that communication channels must be identified to ensure the distribution of information to all concerned levels and parties.

The usefulness of the TSE Roadmap can be seen from the fact that in July 2010 the European Commission published the TSE Roadmap 2. The aim of the new TSE Roadmap is to outline future possible amendments to the current measures to bring them into line with the situation where the EU is finally on the threshold of eradicating BSE in its cattle population. However, amendments to the TSE rules are, and will continue to be made through a step-by-step approach and on a solid scientific basis. In this respect, the scientific advice provided by the European Food Safety Authority will continue to play a crucial role when considering future policy options.

Finally, there is another way to approach the issue of risk research. The last decades have shown that we have reached the limits of a realistic or objective definition of risk, based principally on probabilities (risk equals the seriousness of a hazard multiplied by its probability). This approach does not take sufficient account of the stakeholders involved. The concept of risk can be enriched by taking into account the dimension of perception; this is particularly true in terms of risk communication. Peter Sandman, a risk communication expert, states on his website [http://www.psandman.com] that risk equals hazard plus outrage. Risk communication experts must keep this in mind. In order to minimize outrage, it is important to involve stakeholders in the decision-making process at the earliest possible opportunity. 


\section{References}

Adam, J. 1995. Risk. London: UCL Press.

Bauer, M., Howard, S., Hagenhoff, V., Gasperoni, G. and Rusanen. M. 2006. The BSE and CJD crisis in the press. In C. Dora (Ed.), Health, Hazards and Public Debate: Lessons for Risk Communication from the BSE/CJD Saga (pp. 125-64). Geneva: WHO.

Beck, U. 1986. Risikogesellschaft auf dem Weg in eine andere Moderne [Risk Society: Towards a New Modernity]. Frankfurt: Suhrkamp.

Boyce, C. and Neale, P. 2006. Conducting In-Depth Interviews: A Guide for Designing and Conducting In-Depth Interviews for Evaluation Input. Pathfinder International. Retrieved from http://www.pathfind.org/site/DocServer/m_e_tool_series_indepth_interviews.pdf?docID=6301.

Dien, Y., Dressel, K., Merad, M., Pfeifle, G. and Wright, D. 2006. Final report to the European Commission on STAkeholders in Risk Communication (STARC). Delivered to the European Commission in December 2006.

Dowler, E., Green, J., Bauer, M. and Gasperoni, G. 2006. Assessing public perception: Issues and methods. In C. Dora (Ed.), Health, Hazards and Public Debate: Lessons for Risk Communication from the BSE/CJD Saga (pp. 39-60). Geneva: WHO.

Dressel, Kerstin, Perazzini, Alice; Ru, Giuseppe; Van Wassenhove, Wim (2009): TSE Roadmap - A comparative study of the risk perceptions and risk communications of stakeholders within European countries. Final report to the EC.

Dressel, Kerstin, Ru, Giuseppe, Perazzini, Alice \& Van Wassenhove, Wim (2011): Public risk perception of relaxation of TSE measures in Europe, in: Journal of Toxicology and Environmental Health, Vol. 74, 1. Nov. 2011, pp 1636-1649.

European Commission. 2005. The TSE Roadmap. 322 Final, Brussels, $15^{\text {th }}$ July, 2005.

European Parliament. 1997. Report on alleged contraventions or maladministration in the implementation of community law in relation to BSE, without prejudice to the jurisdiction of the Community and national courts. Part A. Luxembourg.

Funtowicz, S. and Ravetz, J. 1993. The emergence of post-normal science. In R. von Schomberg (Ed.) Science, Politics and Morality: Scientific Uncertainty and Decision Making (pp. 85-123). Dordrecht: Kluwer.

Kermisch, C. 2010. Les paradigmes de la perception des risques [The Paradigms of Risk Perception]. Paris, Lavoisier.

Löfstedt, R. 2003. Risk Communication: Pitfalls and Promises. European Review 11, no. 3: 417-35.

Luhmann, N. 1986. The autopoiesis of social systems. In F. Geyer and J. Van der Zouwen (Eds.) Sociocybernetic Paradoxes: Observation, Control and Evolution of Self-Steering Systems (pp. 172-192). London: Sage.

Maxwell, T.A. 2004. The Public Need to Know: Emergencies, Government Organizations, and Public Information. Government Information Quarterly 20: 233-58.

Public Perceptions of Agricultural Biotechnologies in Europe (PABE) 2001. Final report of the research project funded by the Commission of European communities.

Renn, O. and Benighaus, C. 2006. Framing the perception of cumulative stressors especially chemical risks. Report on approaches to the characterization of knowledge of risks, 
uncertainties and ambiguity and their use and quality assurance in the IP domain. Report of the NoMiracle Project, October 2006.

$\mathrm{Ru}$, Giuseppe, Van Wassenhove Wim, Perazzini, Alice \& Dressel, Kerstin (2010): A comparative study of the risk perceptions and risk communications of stakeholders within five European countries after the issue of the TSE Roadmap, in: Epidémiol. et santé anim., 2010, 57, 13-19.

Slovic, P. 1992. Perception of Risk: Reflections on the Psychometric Paradigm. In S. Krimsky and D. Golding (Eds.), Social Theories of Risk, (pp. 117-152). Westport, CT: PRaeger.

Slovic, P., Fischhoff, B. and Lichtenstein, S. 1982. Why study risk perception? Risk Analysis 2, no. 2: 83-93.

Trettin, L. and Musham, C. 2000. Is Trust a Realistic Goal of Environmental Risk Communication? Environment and Behavior, vol 32, no. 3: 410-426.

Van Wassenhove, W., Dressel, K., Perazzini, A. and Ru, G. 2011. A comparative study of stakeholder risk perception and risk communication in Europe: a bovine spongiform encephalopathy case study. Journal of Risk Research, 2011, 1-18. DOI:10.1080/13669877.2011.646290

Wynne, B. and Dressel, K. 2001. Cultures of Uncertainty - Transboundary Risks and BSE in Europe. In J. Linneroth-Bayer, R. Löfstedt and G. Sjöstedt (Eds), Transboundary Risk Management, (pp. 121-154). London: Earthscan.

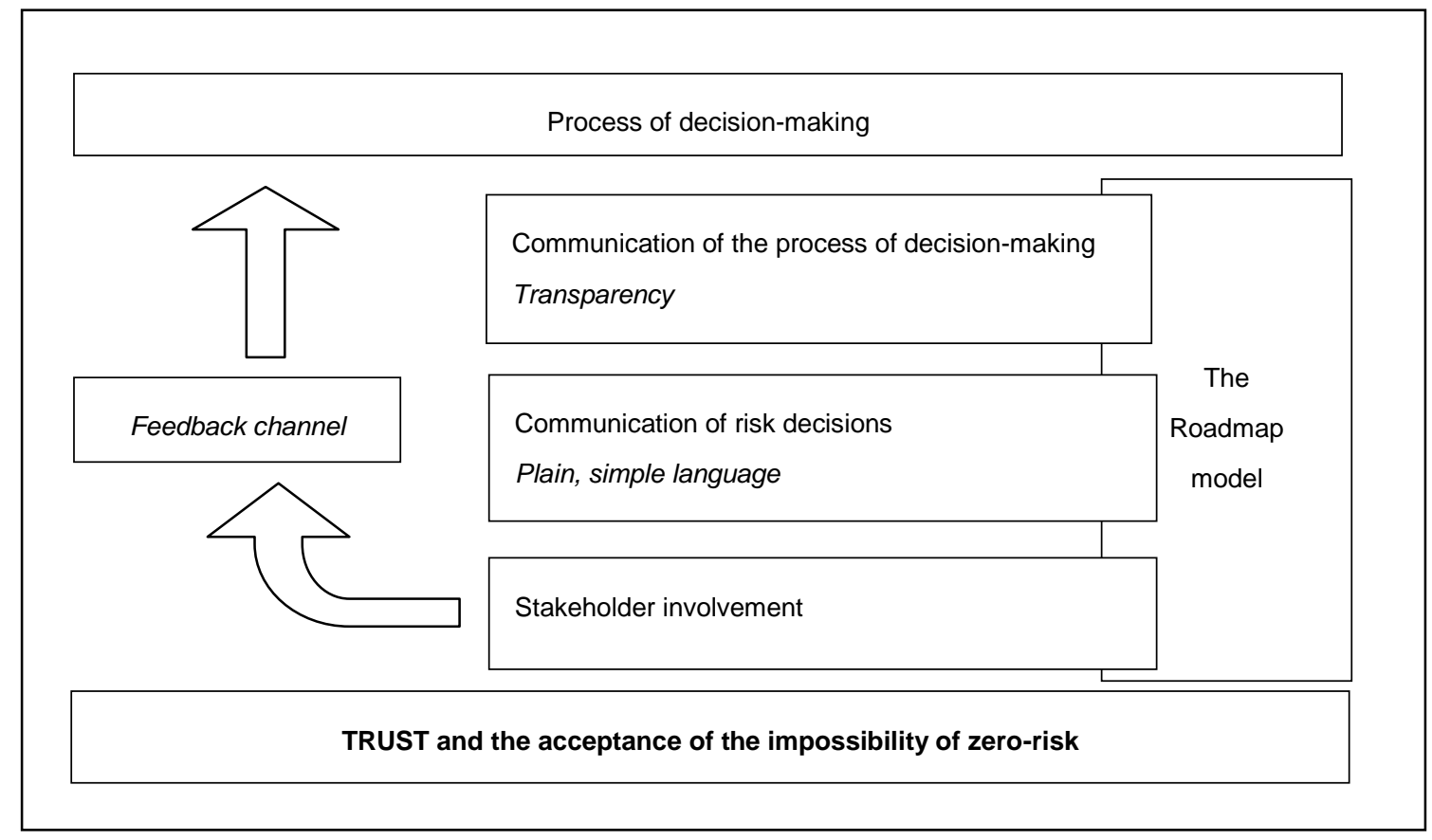

Figure 1: Trust as a by-product of the decision-making process and not as the result of a unilateral act of communication 